\title{
Gossypiboma: An unusual cause of Infected Wound after Inguinal Herniotomy
}

\author{
Naeem Liaqat ${ }^{1},{ }^{*}$ Sajid Hameed Dar ${ }^{2}$, Raja Muhammad Imran ${ }^{1}$, Abeer Asif ${ }^{1}$, Asif Sandhu ${ }^{2}$ \\ 1 Department of Pediatric Surgery, Rawalpindi Medical College, Rawalpindi \\ 2 Department of Pediatric Surgery, SIMS, Lahore
}

\begin{abstract}
Correspondence*: Naeem Liaqat, Assistant Professor Pediatric Surgery, Rawalpindi Medical College, Rawalpindi, Pakistan
E-mail: simsonian.chaudary@gmail.com

(C) 2017, Liaqat et al,

Submitted: 29-06-2017

Accepted: 11-08-2017

Conflict of Interest: None

Source of Support: Nil

This is an open-access article distributed under the terms of the Creative Commons Attribution License, which permits unrestricted use, distribution, and reproduction in any medium, provided the original work is properly cited.
\end{abstract}

\section{DEAR SIR}

Gossypiboma is the term used for inadvertently retained cotton gauze piece into the surgical wound. The most frequent site for gossypiboma is the peritoneal cavity; however, rare locations including neck following thyroid surgery, cranial cavity, scrotum, and urinary bladder have been reported [1-3]. We hereby report a case of gossypiboma following inguinal herniotomy in a child.

A 2-year-old boy presented with inguinal swelling following right sided inguinal herniotomy done 3 months ago in a periphery hospital. The swelling developed just after the surgery and after 2 weeks of surgery, pus started oozing out of the wound. Patient had been taking antibiotics since then but the swelling didn't resolve. On examination, it was slightly tender, tense and non-cystic swelling with oozing of pus when compressed (Fig.1). His x-ray of pelvis showed haziness at right pubic region and differential included osteomyelitis. Ultrasound of the swelling showed it as a mixed echogenicity area, limited to subcutaneous plane and not extending to bone. Per-operatively, pus was present inside the wound and a $(10 \times 10$ $\mathrm{cm}$ ) retained gauze piece was retrieved (Fig.1). Wound was washed with saline and left for secondary healing. Patient was followed up till 3rd week of surgery. His wound healed completely.

Gossypiboma most commonly occurs following laparotomy or thoracic surgical procedures with an incidence of
1 in 3000-5000 intra-abdominal surgeries. But its exact prevalence cannot be determined as it is an under-reported entity [3,4]. Females are more prone for gossypiboma in gynecological procedures [5]. Risk factors may include emergency surgeries, switch over of surgical team or scrub nurse and greater blood loss during surgery [6].

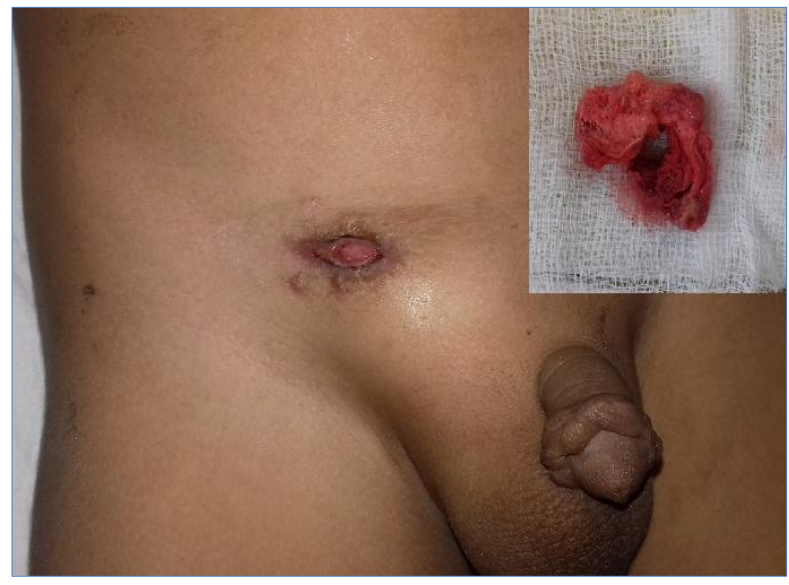

Figure 1: Inguinal swelling with non-healing wound. Inset shows retrieved gauze piece.

Clinical features of retained gauze pieces include wound infection, pus collection in the cavity, intestinal obstruction and peritonitis etc. In our case it was a non-healing infected wound for three months. A case is reported 
where the gauze was retrieved from scrotum after 2 years of surgery [2].

Treatment is surgical exploration and retrieval. Regarding prevention, most important is accurate sponge count and documentation before and at completion of surgery. Also use of radio-opaque gauze pieces, electronic tagging of gauzes, use of Barcodes and use of radiofrequency identification system may be considered [4]. In conclusion, gossypiboma is a completely preventable condition and can be thwarted if operating surgeon is vigilant and principles of safe surgical practice are employed in operation theatre.

Consent: Authors have submitted signed consent form from legal guardians of the patient for use of clinical material in this manuscript. The Consent form is available with Editorial office.

Authors' Contribution: All authors contributed equally in concept, literature review, and drafting of the manuscript and approved the final version of this manuscript.

\section{REFERENCES}

1. Mathew RP, Thomas B, Basti RS, Suresh HB. Gossypibomas, a surgeon's nightmare-patient demographics, risk factors, imaging and how we can prevent it. $\mathrm{Br} \mathrm{J}$ Radiol. 2016;90:20160761.

2. Kretschmer HL VIII. Removal of a gauze sponge from the scrotum, two and a half years after an operation for double inguinal hernia: including a report of five cases in which foreign bodies were left in operative wounds other than in the peritoneal cavity. Ann Surg. 1909;49:814-9.

3. Hussaini MY, Muhammed A, Ahmad B, Hafeez AO, Hycinth MN. Gossypiboma in the scrotum with unusual cutaneous manifestations. Arch Int Surg. 2012;2:33.

4. Gibbs VC, Coakley FD, Reines HD. Preventable errors in the operating room: retained foreign bodies after surgerypart I. Curr Probl Surg. 2007;44:281-337.

5. Mavrigiannaki P, Dastamani C, Vouza E, Lambropoulou E, Kairi-Vassilatou E, Kondi-Pafiti A. Gossypiboma: a rare abdominal lesion of women after cesarean section, usually misdiagnosed as a neoplasm. Clin Exp Obstet Gynecol. 2010;38:294-6.

6. Gawande AA, Studdert DM, Orav EJ, Brennan TA, Zinner MJ. Risk factors for retained instruments and sponges after surgery. N Engl J Med. 2003;348:229-35. 